\title{
ERS statement on the multidisciplinary respiratory management of ataxia telangiectasia
}

\author{
Jayesh M. Bhatt ${ }^{1}$, Andrew Bush ${ }^{2}$, Marjo van Gerven ${ }^{3}$, Andreea Nissenkorn ${ }^{4,5}$, \\ Michael Renke 6 , Lian Yarlett ${ }^{7}$, Malcolm Taylor ${ }^{8}$, Thomy Tonia $^{9}$, Adilia Warris ${ }^{10}$, \\ Stefan Zielen ${ }^{6}$, Shairbanu Zinna ${ }^{1}$ and Peter J.F.M. Merkus ${ }^{3}$
}

\begin{abstract}
Affiliations: ${ }^{1}$ Nottingham Children's Hospital, UK Paediatric National Clinic, Nottingham University Hospitals NHS Trust, Nottingham, UK. ${ }^{2}$ Imperial College and Royal Brompton Hospital, London, UK. ${ }^{3}$ Dept of Paediatrics, Division of Respiratory Medicine, Amalia Children's Hospital Radboud, University Medical Centre, Nijmegen, The Netherlands. ${ }^{4}$ Rare Diseases Service and Pediatric Neurology Unit, Edmond and Lilly Safra Pediatric Hospital, Sheba Medical Center, Tel HaShomer, Israel. ${ }^{5}$ Sackler School of Medicine, Tel Aviv University, Tel Aviv, Israel. 'Dept of Allergology, Pneumology and Cystic Fibrosis, Children's Hospital, GoetheUniversity Theodor-Stern Kai, Frankfurt/Main, Germany. ${ }^{7}$ The A-T Society, IACR-Rothamsted, Harpenden, UK. ${ }^{8}$ School of Cancer Sciences, University of Birmingham, Birmingham, UK. ${ }^{9}$ Institute of Social and Preventive Medicine, University of Bern, Bern, Switzerland. ${ }^{10}$ Institute of Medical Sciences, University of Aberdeen and the Royal Aberdeen Children's Hospital, Aberdeen, UK.
\end{abstract}

Correspondence: Jayesh M. Bhatt, Nottingham Children's Hospital, Respiratory Paediatrics, East Block, QMC, Derby Road, Nottingham, NG7 2UH, UK. E-mail: jayeshbhattlahotmail.com

ABSTRACT Ataxia telangiectasia (A-T) is a rare, progressive, multisystem disease that has a large number of complex and diverse manifestations which vary with age. Patients with A-T die prematurely with the leading causes of death being respiratory diseases and cancer. Respiratory manifestations include immune dysfunction leading to recurrent upper and lower respiratory infections; aspiration resulting from dysfunctional swallowing due to neurodegenerative deficits; inefficient cough; and interstitial lung disease/ pulmonary fibrosis. Malnutrition is a significant comorbidity. The increased radiosensitivity and increased risk of cancer should be borne in mind when requesting radiological investigations. Aggressive proactive monitoring and treatment of these various aspects of lung disease under multidisciplinary expertise in the experience of national multidisciplinary clinics internationally forms the basis of this statement on the management of lung disease in A-T. Neurological management is outwith the scope of this document.

0

@ERSpublications

Complex respiratory manifestations in ataxia telangiectasia require regular, proactive multidisciplinary management http://ow.ly/Solqe

\section{Introduction}

Ataxia telangiectasia (A-T) (Louis-Bar syndrome; OMIM 208900) is an autosomal recessive disorder caused by mutations in the gene ATM (ataxia-telangiectasia mutated) (11q22.3). This gene is expressed ubiquitously and encodes a protein kinase (ATM kinase) which plays a key role in the control of double-strand-break DNA repair. The Orphanet registry estimates the average prevalence of A-T at 1/100000 children [1]. The most reliable estimates for the number of people with A-T, in the UK at least, are 3 per million [2], and hence individual clinicians are likely to have limited experience in treating the disease. The prevalence of patients with A-T in Europe is estimated to be 1/150000 [3]. In Europe, 410 living A-T patients are currently

For editorial comments see Eur Respir J 2015; 46: 1557-1560 [DOI: 10.1183/13993003.01456-2015].

Received: Sept 012015 | Accepted after revision: Sept 082015

Conflict of interest: None declared.

Provenance: Submitted article, peer reviewed.

Copyright CERS 2015. ERR articles are open access and distributed under the terms of the Creative Commons Attribution Non-Commercial Licence 4.0. 
listed on the European Society for Immunodeficiencies registry [4]. A clinician faced with a patient or family with a child with A-T will want to access the requisite experience, expertise, advice and ongoing research, and to make appropriate referrals. It is important that patients and professionals obtain appropriate expert advice and recommendations on the management of this rare condition.

$\mathrm{A}-\mathrm{T}$ is a progressive, multisystem disease that has a large number of complex and diverse manifestations that vary with age (table 1$)$.

The clinical picture of this condition can be very variable and the severity of the pulmonary, immunological and neurological manifestations varies widely between patients and is related to the severity of the underlying mutations and any residual ATM kinase activity [5]. It has been recently suggested that the name A-T should be replaced by ATM syndrome. ATM syndrome represents a neurodegenerative disorder with multisystem involvement due to the absence or reduced levels of ATM protein and kinase activity [6]. The syndrome is characterised by the presence of movement disorders, such as cerebellar ataxia, dystonia, chorea and myoclonus, in association with systemic abnormalities such as immunodeficiency, malignancies, oculocutaneous telangiectasias and an increase in $\alpha$-fetoprotein levels. The disease most commonly presents with ataxia during the third or fourth year of life. The important first step in the evaluation of young children presenting with ataxia should be $\alpha$-fetoprotein testing. The diagnosis should then be confirmed by genetic testing to identify the mutations and measure the product of the ATM gene, the protein kinase ATM. This diagnostic test is likely to be available in specialised laboratories only.

Patients with A-T die prematurely, the leading causes of death being respiratory diseases and cancer. A minimally estimated annual mortality rate for white patients is $19.5 / 1000$ for ages $15-19$ years and reportedly three-fold higher for African-American patients [7]. In two large cohorts of patients with A-T, one prospective (median age at death estimated by a modification of the Kaplan-Meier survival curve) and one retrospective, median survival was 25 years and 19 years, respectively. There was a wide range of age at death. Mortality crossed the 25 th and 75 th centiles in the prospective cohort at ages 18 years and 28 years, and at ages 14 years and 28 years in the retrospective cohort. In these two cohorts, life expectancy did not correlate well with severity of neurological impairment [8]. The large French registry study showed that the prognosis for patients with A-T has not changed since 1954, with the Kaplan-Meier 20-year survival rate reported as 53.4\% [9].

\section{Scope of the article}

This article examines the respiratory aspects of $\mathrm{A}-\mathrm{T}$, including chronic and recurrent infections; bronchiectasis and interstitial lung disease (ILD); immunodeficiency associated with A-T; and aspiration

\section{TABLE 1 Clinical manifestations of ataxia telangiectasia}

Respiratory

Immunodeficiencies

Increased risk of malignancy

Neurological"

Other"
Recurrent respiratory tract infections including otitis media, sinusitis, bronchitis and pneumonia

Bronchiectasis secondary to recurrent/chronic infection and aspiration Interstitial lung disease

Obliterative bronchiolitis

Aspiration syndromes due to incoordinate swallowing

Opportunistic infections

Restrictive lung disease due to scoliosis, neuromuscular disease or fibrosis

Clinically not apparent in all patients

Variable degrees

Generally nonprogressive

$\lg A$ and $\lg G_{2}$ deficiency most common

Low total IgG and IgE also seen

Poor polysaccharide antibody responses

Variable cellular immunodeficiency (low numbers of T- and B-cells)

Mainly lymphoid tumours (in patients aged $<16$ years)

Lymphoid and nonlymphoid tumours in older patients

Neurodegeneration of the cerebellum

Progressive cerebellar ataxia

Oculomotor apraxia

Movement disorders: chorea, dystonia

Ocular, cutaneous and mucosal telangiectasias

Accelerated ageing

Diabetes mellitus

\#: there are no pulmonary telangiectasias; ": not reviewed in this article. 
syndromes due to incoordinate swallowing. The management of the ataxia itself and developmental delay is outside the scope of the article.

We aim to categorise the phenotypes of lung disease, examine the natural history and describe current best-practice monitoring and treatment strategies for various aspects of lung disease. There are no randomised controlled trials with respect to the treatment of the respiratory complications in A-T. This paper serves as a consensus statement which summarises current evidence and best practice, and aims to set out the future translational, epidemiological and clinical research agenda. Accordingly, we are not able to formally evaluate the evidence using GRADE (Grading of Recommendations Assessment, Development and Evaluation) (http://taskforces.ersnet.org/about-task-forces/item/how-to-apply-for-ers-funding).

\section{Methods}

This multidisciplinary European Respiratory Society (ERS) task force comprises a broad range of experienced clinicians (respiratory, infectious diseases, immunology, neurology and genetics fields, physiotherapists and a speech and language therapist) as well as a patient representative from the national UK patient charity for A-T (L. Yarlett, who is the parent of a young person with A-T and chairman of the board of trustees of the A-T Society). This document was created by combining a firm evidence-based approach where available and the clinical expertise of the task force members. However, a formal grading of the evidence was not performed, and therefore, this document does not contain recommendations for clinical practice. The process adopted for this statement was agreed by all task force members. Each member was requested to frame important clinical questions for the management of lung disease in A-T. The literature searches were carried out by S. Zinna based on the questions framed and they covered the years from 2000 to 2015. In addition, the references of the selected papers were scrutinised for further relevant evidence. Hand searches of A-T clinical research conference abstract books were made by S. Zinna and J. Bhatt. Publications that were in print in English were selected for further examination. Observational studies (no published systematic reviews or randomised controlled trials) from MEDLINE/ PubMed, Embase, the Cochrane Central Register of Controlled Trials, CINAHL (Cumulative Index to Nursing and Allied Health Literature) and the Cochrane Collaboration were collected. The scientific evidence relevant to the delegated subject area was reviewed. Results were discussed by all members of the task force. The final document was written by the chairs of the task force ( J. Bhatt and P. Merkus). The draft manuscripts were reviewed by all task force members to ensure appropriateness and relevance, and the final document was accepted by all task force members.

Phenotypes of respiratory disease in A-T

Aetiology, natural history and physical examination

There are three recognised major lung disease phenotypes. 1) Immune dysfunction leading to recurrent upper and lower respiratory tract infections (RTIs), which in turn can lead to bronchiectasis; 2) lung disease associated with dysfunctional swallow and inefficient cough due to neurodegenerative deficits, which increases the risk for aspiration and consequently may also cause or worsen bronchiectasis; and 3) ILD/pulmonary fibrosis.

There is a significant overlap in the initial presenting symptoms of the phenotypes described above. For example, chronic or intermittent chest congestion with a wet/moist cough may be due to cough impairment with difficulty clearing airway secretions, or related to infections secondary to immune deficiency, but could also be due to aspiration. In contrast, ILD usually causes a dry cough with associated tachypnoea, crackles, hypoxaemia and finger clubbing in the absence of viral or bacterial infections.

\section{Immune deficiency}

This is multifactorial, with both reduction of circulating T- and B-cell numbers (caused by reduced thymic output and increased apoptosis) and abnormal lymphocyte function (restricted antigen receptor repertoires, abnormal B-cell class switching and disturbed naive B- and T-cell homeostasis) contributing to the clinical phenotype $[10,11]$. The variable severity of antibody deficiency is related to disturbed naive B- and T-cell homeostasis, probably because of reduced B- and T-cell production linked to disturbed V(D)J gene segment recombination, and consequently a limited $\mathrm{B}$ - and $\mathrm{T}$-cell receptor repertoire. There is also a reduction of T-cell-dependent memory B-cell populations [11]. The most common humoral immunological defects are markedly diminished or absent serum $\operatorname{IgA}$ and $\mathrm{IgG}_{2}$, and impaired antibody responses to vaccines [12]. Serum IgG level can be normal even when some IgG subclasses are reduced, although a subset of classical A-T patients $(\leqslant 10 \%)$ will have a severe early-onset hypogammaglobulinaemia [13] and increased IgM levels [14]. The A-T patients with increased serum IgM levels show a remarkably more severe disease course [12]. Patients with $\operatorname{IgG}_{2}$ or $\operatorname{IgG}_{4}$ deficiency appear to be at a higher risk of RTIs [15]. The patients with classical A-T generally have two truncating mutations resulting in total absence of ATM kinase activity, while patients with milder phenotypes have at least one missense or splice site mutation resulting in the expression of ATM 
with some kinase activity (see the section on phenotype/genotype correlation). Patients with variant A-T have a later onset of disease and less severe antibody deficiency $[5,10,11]$.

Cellular immunity can also be affected in A-T: abnormal development of the thymus, with absence of Hassall's corpuscles and corticomedullary differentiation, impaired delayed hypersensitivity reactions or lymphoproliferative responses to mitogens and diminishing numbers of CD4 lymphocytes [16]. T-cell lymphopenia is a commonly observed laboratory finding in people with A-T, but its clinical significance remains unclear, and the fact that $\mathrm{A}-\mathrm{T}$ patients do not show susceptibility to classical opportunistic infection, for example with Pneumocystis jirovecii, remains poorly understood.

\section{Response to immunisations}

Routine childhood immunisations, including attenuated live viral vaccines, would usually have been given prior to the diagnosis of A-T and/or recognition of associated immunodeficiency [12]. Reports of adverse events in patients with $\mathrm{A}-\mathrm{T}$ after the administration of live vaccines are rare, and for the most part vaccines are well-tolerated [17].

Patients with A-T are capable of making protective antibodies to routine childhood (conjugated) vaccines such as diphtheria and tetanus, but the response to the 23 -valent pneumococcal polysaccharide vaccine (PPV) appears to be variable. NOWAK-WEGRZYN et al. [12] reported that $69 \%$ of patients showed protective levels of antibody for all tested serotypes in response to PPV, while other studies have reported poor antibody response to this immunisation $[18,19]$. Immune response to the 7-valent pneumococcal conjugate vaccine (PCV) appears to be sufficient, with two- to 7.5-fold increases over baseline after immunisation for all seven serotypes [20]. A follow-up booster dose with PPV after PCV seems to augment the response to antibody levels to the PPV23, though the increases in pneumococcal antibody titres from baseline were lower (1.5- to seven-fold increase) in A-T patients than controls (nine- to 34-fold increase) [19].

Inactivated influenza vaccine is usually given to all patients with A-T and their close family contacts; there are no data available on the live attenuated nasal influenza vaccine, but as mentioned above other live attenuated viral vaccines have been tolerated in children with A-T [12]. This is usually offered even to those prescribed immunoglobulin replacement therapy, as the administered immunoglobulin may not contain specific antibodies against the antigens of the seasonal influenza viruses [16]. Varicella zoster vaccine $(\mathrm{VZV})$ is also offered to those who have not already experienced chickenpox and are nonimmune and in those who are not profoundly immunocompromised. Post-exposure prophylaxis with VZV immunoglobulin and aciclovir are usually considered in those who are profoundly immune-suppressed [16]. Human papilloma virus vaccine is an inactivated vaccine, which on theoretical grounds is particularly important for girls with $\mathrm{A}-\mathrm{T}$ in view of the apparent abnormal response to papilloma viruses causing cutaneous warts, and the general predisposition to malignant disease including carcinomas. Bacille Calmette-Guerin vaccine is not used routinely, but can be considered for use in those patients with an increased risk of tuberculosis exposure (depending on geographical area and origin of parents from tuberculosis-endemic countries), unless there is profound T-cell lymphocytopenia [21].

\section{Laboratory abnormalities and clinical infections}

NowAK-WEGRZYN et al. [12] found no correlation between frequency of RTIs and deficiency of IgG, IgA or subclasses of IgG.

\section{Types of infections}

Several retrospective case series (both published [8, 15, 20, 22] and unpublished (S. Zielen, Dept of Allergology, Pneumology and Cystic Fibrosis, Children's Hospital, Goethe-University Theodor-Stern Kai, Frankfurt/Main, Germany; personal communication)) have shown a high prevalence of recurrent and chronic upper and lower RTI, the latter becoming more prevalent with increasing age. Definitions of recurrent infections did vary in these reports, e.g. recurrent respiratory infections as five or more respiratory manifestations (other than pneumonia) per year or one episode of pneumonia in a year [23] or more than three respiratory infections per winter season requiring antibiotics or a severe/atypical response to a single infection [10]. Preliminary results from a small prospective study found highly significant differences for children with A-T (median (range) 11 (2-25) years) and age-matched controls in symptom scores, number of episodes, days with symptoms and missed days of kindergarten, school or work. However, severe infections with hospitalisation were surprisingly low in prevalence, but prolonged cough after viral infections was frequently present in patients with A-T (S. Zielen; personal communication).

Radiologically confirmed bronchiectasis seems to be established by the end of first decade $[9,17,23]$. The prevalence of bronchiectasis varied from $10 \%$ [17] to $47 \%$ [23] in these series. The experiences of members of the task force suggest that proactive monitoring and aggressive multidisciplinary treatment delays the onset or stabilises bronchiectasis. 


\section{Microbiology of lung disease in A-T}

Not all studies had microbiological confirmation of infection $[9,10,12,22]$, and even when this was available, the nature of the samples obtained was heterogeneous (nasopharyngeal aspirate, throat swabs, cough swabs, sputum or bronchoalveolar lavage or serological confirmation on blood tests) [17, 23, 24]. Sputum microscopy and culture in children who can cough and produce sputum [23] and cough swabs in those who cannot [24] are usually carried out at each visit. Sputum induction in nonexpectoring patients or bronchoalveolar lavage (which, being invasive, is a last resort) are sometimes required in children with troublesome respiratory symptoms but negative microbiology results [23].

\section{Viruses}

Respiratory infections are common in A-T during the early childhood years $[12,23]$ although the overlap with normal age-related susceptibility to early viral infections can be difficult to tease out. The common respiratory viruses can be detected even at scheduled nonurgent clinic visits [24]; however the role and impact of viral infections in A-T remain to be determined.

\section{Bacteria}

Pulmonary infections in A-T are usually caused by common bacterial pathogens in later childhood, such as Haemophilus influenzae, Streptococcus pneumoniae, Pseudomonas aeruginosa and Staphylococcus aureus. The airways of younger patients with A-T were found to be more likely to be colonised with $S$. aureus, $H$. influenzae or $S$. pneumoniae, and over time, these colonising pathogens were replaced by mucoid $P$. aeruginosa $[12,17,23,24]$.

\section{Opportunistic infections}

These are uncommon in patients with A-T. Most reported series have found no evidence of any opportunistic pathogens and even when reported, they are uncommon $[9,12,17,24]$.

The microbiology of the respiratory tract in patients with A-T is thus unlike that of other primary immune deficiencies and has more similarities to that seen in patients with cystic fibrosis [17]. Other similarities to cystic fibrosis are that systemic spread of infectious organisms is rare, opportunistic infections are not observed and bronchiectasis and pneumothoraces are common results of recurrent pulmonary insults $[17,23]$.

\section{Does immune deficiency progress?}

The immune system in A-T is probably abnormal from birth. There have been only a few reports of patients with progressive immunodeficiency $[25,26]$. A retrospective study [12] found no difference in the prevalence of Ig deficiencies over time. A-T patients do not appear to have increased frequency or greater severity of immune abnormalities with age and, in general, for the majority of patients the severity of immune deficiency (both clinically and in terms of immunological blood markers [11]) does not seem to deteriorate significantly. If recurrent respiratory tract infections present later in life, then other contributory factors (e.g. cough/swallowing difficulties, neuromuscular abnormalities or underlying lung disease) are a more likely explanation [27].

\section{Immune replacement and prophylactic antibiotic therapy}

No placebo-controlled trials have been performed with regards to prophylactic antibiotic therapy or immune replacement therapy.

\section{Antibiotics}

RTIs affect patient survival and are an independent risk factor for mortality in A-T. There is a large variation in the practice of prescribing prophylactic antibiotics for patients with A-T. A large registry study [9] suggested that antibiotic prophylaxis (effective against encapsulated bacteria, such as H. influenzae and $S$. pneumoniae) might be beneficial at early stages of the disease, but this is based on an extrapolation from other immunodeficiency diseases. A high proportion (74\%) of children with A-T are prescribed prophylactic antibiotics [24] on inconsistent and non-evidence-based criteria. A longitudinal follow-up study (mean duration of follow-up 5 years) found that 30 (68\%) out of 44 patients were commenced early on prophylactic antibiotics with an aim to reduce the frequency and severity of bacterial respiratory infections [27]. The proportion of patients with A-T who had chronic respiratory symptoms and were using chronic macrolide antibiotics was lower in the patients in the USA (12 out of $36 \mathrm{~A}-\mathrm{T}$ patients alive and 0 out of $65 \mathrm{~A}-\mathrm{T}$ patients who died secondary to lung disease); however comparative data for patients with A-T who did not have chronic respiratory symptoms were not available [17].

There are no controlled trials that provide evidence of eradication of $P$. aeruginosa colonisation/infection [24] in A-T. Anecdotally and using the approach taken in cystic fibrosis, a combination of oral 
ciprofloxacin and nebulised colistin seems to be favoured by clinicians. In those with more advanced disease, combination intravenous antipseudomonal antibiotics are often used.

Prophylactic antibiotic treatment is considered on an individual basis when the burden of respiratory infections is high, but microbiological resistance and side-effects should be always borne in mind.

\section{Immunoglobulin replacement}

The decision to commence Ig replacement therapy is taken on clinical grounds in those children who have a history of recurrent respiratory infections and low specific antibody responses despite booster immunisations [16]. Patients with borderline low serum IgG concentrations for age but protective antibody responses to childhood vaccines and no history of severe or recurrent infections do not need to be on replacement therapy [12]. Hence there is wide variation in the proportion of patients who are on replacement treatment (table 2).

In the series from the USA [12] the median (range) age at initiation of i.v. Ig therapy was 4 (1.9-10.7) years. Recurrent respiratory infections decreased in frequency, duration and severity [12, 23] and all children reported that i.v. Ig increased their general wellbeing [23]. Supplemental Ig administration in individuals with A-T was associated with lower forced vital capacity (FVC) \% predicted. This association may be due to a greater frequency of RTIs in those individuals with more severe immunodeficiency, thus accounting for their lower pulmonary function. This is a clear bias since the more severe patients are treated with i.v. Ig [28], as was also the case in the series by NoorDZI et al. [13], which included A-T patients with hyper-IgM and severe immune deficiency. These findings suggest that the recognition of groups who may be at higher risk of lower pulmonary function may help to direct care and improve clinical outcomes in A-T. If Ig therapy is considered, most centres administer subcutaneous Ig, as is currently recommended for severe immundeficiencies [29-31].

\section{Interstitial lung disease}

ILD was present in $25(25.8 \%)$ out of 97 patients with A-T who had either chronic respiratory symptoms or pulmonary disease listed as cause of death [32]. None of these patients had evidence of an infectious process preceding the onset of their lung disease. Many of them had been previously treated for cancer. The mean (range) age of onset of ILD was 17.5 (9-28) years. The signs and symptoms that preceded radiographic evidence of ILD were a nonproductive cough in 21 out of 25 patients, dyspnoea in 12 out of 25 patients and fever in 11 out of 25 patients. All 10 patients who presented with a cough of $>1$ month's duration, dyspnoea, fever and abnormal auscultatory changes had interstitial changes on chest radiography. None had lasting clinical improvement after treatment with antibiotics. ILD could be present even in the absence of overt immune deficiency. The histological pattern of A-T ILD does not fit a specific American Thoracic Society/ERS category among idiopathic interstitial pneumonias, and may represent a unique class of ILD. The ILD of A-T is distinctive in its histology, which is uniquely characterised by lung fibrosis and chronic inflammation with lymphocytic or lymphohistiocytic cells, and the presence of atypical epithelial and interstitial cells with large hyperchromatic and pleiomorphic nuclei. Bronchoalveolar lavage fluid contains $>70 \%$ lymphocytes, $20 \%$ macrophages, $<5 \%$ neutrophils and no eosinophils or neoplastic cells [32]. The response to corticosteroids is discussed later [32].

Clinical examination often does not contribute to the diagnosis of ILD, as signs and symptoms are nonspecific. Restrictive lung disease on pulmonary function testing may suggest the presence of an interstitial process. A chest radiograph scan (fig. 1) is usually considered justifiable for any A-T patient with pulmonary symptoms that do not remit fully after vigorous treatment of infection, because the consequences of not imaging are much less than any concern that the patient may suffer tissue damage

\section{TABLE 2 Replacement Ig replacement therapy in ataxia telangiectasia}

\begin{tabular}{lcc} 
Study design [ref.] & Subjects $\mathbf{~}$ & Subjects receiving Ig therapy (\%) \\
\hline Retrospective [10] & 80 & $12.5^{\#}$ \\
Retrospective [12] & 100 & 13 \\
Retrospective [23] & 15 & 60 \\
Retrospective [9] & 240 & $34.8^{\text {巾 }}$ \\
Retrospective [27] & 44 & 15.9 \\
Retrospective [28] & 100 & 18.8
\end{tabular}

${ }^{\#}: 17 \%$ of those with null mutations; ${ }^{\text {I: }} 75.6 \%$ of the patients with bronchiectasis. 


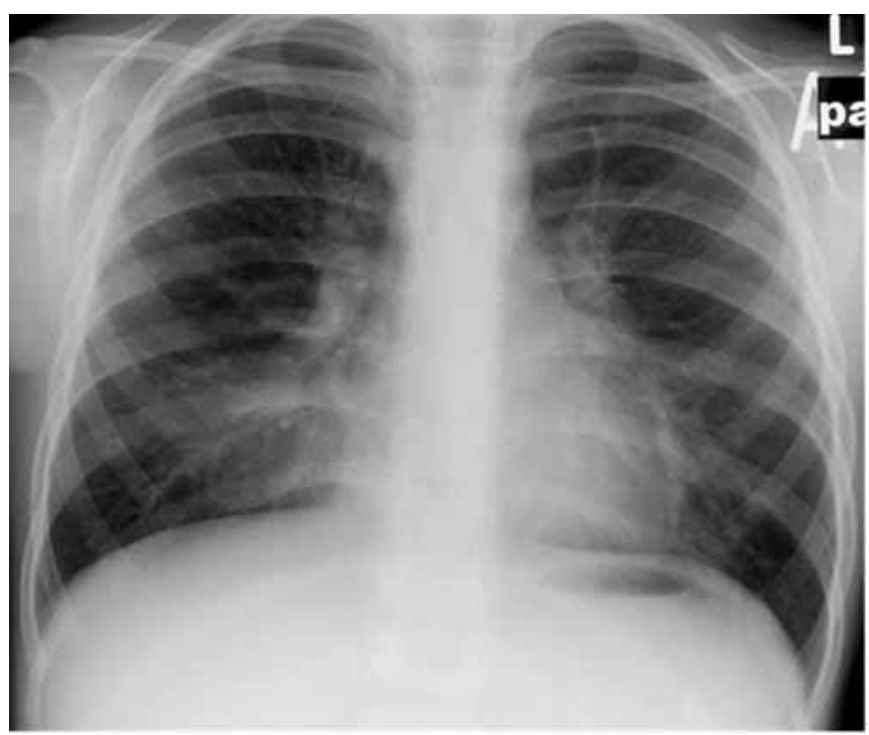

FIGURE 1 A plain chest radiograph of a patient with ataxia telangiectasia showing diffuse bibasal interstitial changes.

from this dose of irradiation. Similarly, even high-resolution computed tomography (CT) (fig. 2) is usually considered justifiable (although magnetic resonance imaging (MRI) could become the technique of choice, as discussed later) as a prelude to a lung biopsy to confirm the diagnosis of ILD. However, the diagnostic benefits of a procedure such as a lung biopsy should be weighed against the risks associated with anaesthesia and surgery (see the later section on diagnostic and therapeutic radiation).

The aetiology and pathogenesis of ILD and pulmonary fibrosis in A-T patients is unknown. Various potential triggers/contributors including atypical or viral infections and chronic aspiration, as well as the effect of immune deficiency itself (ILD is well described in immune deficiency, albeit more typically as part of the follicular bronchiolitis-lymphoid interstitial pneumonitis picture) may play a role. In the largest reported series [32] no correlation was found between the development of ILD and abnormalities in Ig levels. Those patients given chemotherapy appeared to have a different type of ILD with pulmonary fibrosis dominating.

Only treatment with systemic corticosteroids early in the course of the illness was associated with clinical and radiographic improvement. 19 out of 25 patients with ILD died within 24 months of disease onset. No

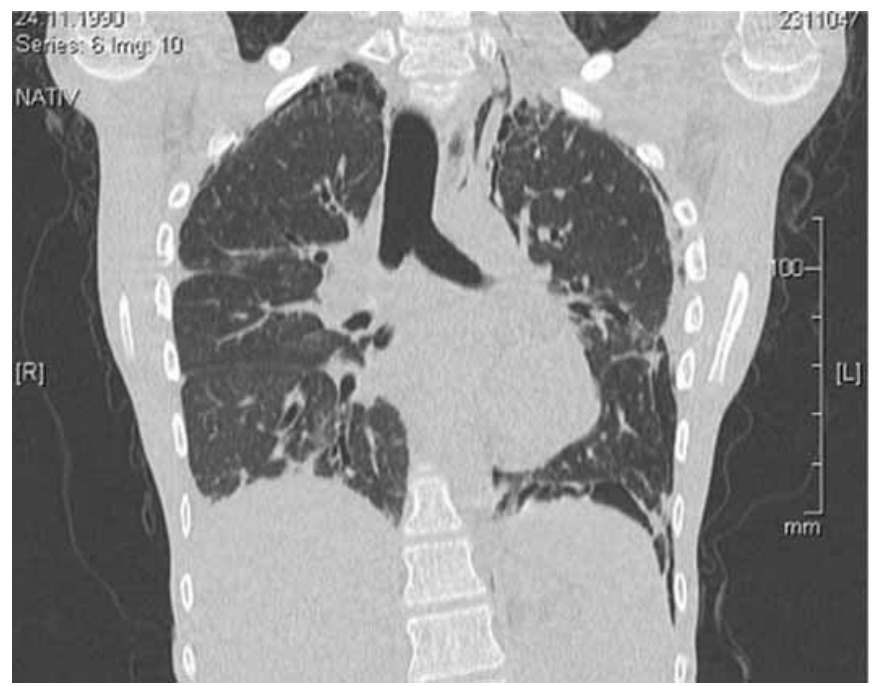

FIGURE 2 Chest computed tomography scan of a patient with ataxia telangiectasia showing diffuse bibasilar interstitial and interlobular reticular opacities with interlobular septal thickening, and bronchiectasis in the right lower lobe. Note that any radiological imaging should use the minimum possible doses, and concerns about radiosensitivity need to be addressed. 
patient who commenced treatment with systemic corticosteroids $>1$ year after the onset of interstitial changes was alive, while five out of seven patients treated early with corticosteroids were alive at the time of evaluation. Hence, a high degree of suspicion is warranted so that those patients with ILD can be treated early and aggressively. The benefits of treatment with corticosteroids needs to be weighed up against potential side-effects including inducing or aggravating diabetes or osteoporosis. The latter is probably secondary to poor nutrition and early confinement to wheelchair. There is animal experimental evidence that ATM deficiency leads to an osteoporotic phenotype in mice due to reduced bone formation and increased bone resorption [33] or accelerated cellular ageing [34].

Other noninfective causes of diffuse lung diseases (which may mimic ILD) in A-T include pulmonary parenchymal involvement secondary to lymphoma [35, 36] and pulmonary fibrosis secondary to chemotherapy for the treatment of a malignancy [37]. The risk of rapid pulmonary decline following the use of chemotherapeutic agents such as bleomycin is well described in A-T and may be due to an underlying defect in injury repair [38]. These agents are used with great caution in A-T patients, if at all, as they appear to have the same effect on cultured A-T homozygous cells as ionising radiation [39]. Lymphocytic interstitial pneumonitis has been reported as another cause of diffuse lung disease in A-T and this responds to glucocorticosteroid treatment [40].

\section{Respiratory monitoring}

The underlying neurological involvement [41] can make it difficult to obtain reliable reproducible dynamic lung function tests that require any effort and coordination. However, several researchers have demonstrated that pulmonary function testing with certain modifications can be performed reliably and reproducibly by patients with A-T so that it may be used to track the rate of decline in lung function over time $[23,28,41-43]$.

\section{Standard spirometry}

The reproducibility of testing is increased by stabilising the patient's head and holding the cheeks while the patient performs the forced expiratory manoeuvre, optimising the fit of the mouthpiece and assessing for leaks in the system, and thus the majority of A-T adolescents are able to perform spirometry with minimal modifications [41]. Flow-volume curves do meet recommended reproducibility criteria but often fail to meet other standard recommendations for forced spirometry [43] and will often underestimate respiratory function.

McGrath-Morrow et al. [41] found that most adolescents tested had normal functional residual capacity (FRC), near-normal total lung capacity, high residual volume (RV), decreased vital capacity and low maximal expiratory pressure, suggesting either or both expiratory muscle weakness and incoordination and difficulty expiring to RV. Since the measurement of FRC requires only passive cooperation, they considered this the most reliable lung measurement in individuals who may not be able to perform maximal inspiratory and expiratory manoeuvres. As the vital capacity \% pred values did not correlate with FRC \% pred values, the decreased vital capacity \% pred values in the A-T adolescents may represent difficulty with complete expiration (i.e. due to muscle weakness or coordination problems) rather than small lung volumes. Since FVC predicted values in A-T are often low, the interpretation of sequential spirometry may best be performed relative to an individual's baseline study rather than compared to normal predicted values [43]. BERKUn et al. [42] studied a younger cohort of children with A-T (median (range) age 8.8 (3.7-19.3) years). They found that the rise time to peak flow was three to four times longer than that of healthy values in most of their patients (250-350 ms versus $70-80 \mathrm{~ms}$ in the healthy population). This delay in initiation of the breath may not be due to obstructive lung disease per se, but in part secondary to neurological dysfunction and delayed processing before initiating the expiratory breath. As (young) A-T patients often do not exhale for more than $1 \mathrm{~s}$, forced expiratory volume in $1 \mathrm{~s}\left(\mathrm{FEV}_{1}\right)$ may not be an accurate parameter for describing bronchial obstruction in this particular group and FEV0.5 may be a better index [43].

Obstructive and mixed patterns are found in a small proportion of people with A-T [23, 28, 41, 42]. When a reproducible obstructive pattern is observed on baseline flow-volume loops, bronchodilator reversibility is assessed to determine whether bronchodilators and possibly inhaled corticosteroids would be beneficial. Impulse oscillometry, a noninvasive test that can measure airway resistance, is an option in children who are unable to perform spirometry reliably.

The possible causes of an obstructive pattern include suboptimal lung function testing through poor coordination while breathing; bronchiectasis; small airways involvement due to infections with or without aspiration; and asthma or other coincidental airways disease.

Inability to expire fully to RV and a short expiratory time may result in a decreased FVC, a high FEV1/FVC ratio and the appearance of restrictive lung disease. If a restrictive pattern is suspected, this requires 
confirmation from assessment of total lung capacity and other static lung volumes (using body plethysmography or a gas dilution technique). However, as many patients are dependent on wheelchairs and may be unable to perform the required respiratory manoeuvres, body plethysmography is often not a practical option. If available, helium dilution provides an effort-independent alternative for measuring lung volumes [44].

The possible causes of restrictive patterns include suboptimal lung function testing through poor coordination while breathing, muscle fatigue, inspiratory muscle dysfunction, ILD and scoliosis or other chest wall deformity.

McGrath-Morrow et al. [28] explored several potential factors associated with lower lung function in A-T. They found that females aged $\geqslant 11$ years, males who had higher chromosomal breakage and the need for supplemental $\gamma$-globulin were associated with lower FVC \% pred, but body mass index (BMI) percentile or gastric tube placement for nutritional supplementation were not. Some reassurance is offered by the observation that in a subset of 25 people who underwent pulmonary function testing on more than one occasion over an average of 2 years, there was no significant decline.

\section{Gas exchange}

Diffusing capacity of the lung for carbon monoxide (DLCO) measurements are sometimes considered, particularly to provide evidence of impaired gas exchange when evaluating a patient for ILD. However, advanced A-T disease is associated with severe neurodegenerative dysfunction and such tests, especially the single-breath DLCO measurement may simply not be feasible; measurement of DLCO by rebreathing technique may be required. Therefore, there are arguments for monitoring alternative end-points closely, such as clinical condition (respiratory rate and effort and auscultation), transcutaneous oxygen saturation at rest (and during exercise or overnight oxygen saturations if possible).

Young adults with more advanced lung disease are monitored for worsening type 2 respiratory failure by assessing for hypercapnia by either capillary blood gases or end-tidal carbon dioxide monitoring. Chronic respiratory insufficiency was present in eight children (mean $\pm \mathrm{SD}$ arterial oxygen tension $78 \pm 5 \mathrm{mmHg}$ ) at a mean age of 10 years. Alveolar hypoventilation was also diagnosed in two patients (mean arterial carbon dioxide tension <52 mmHg; normal range 35-45 $\mathrm{mmHg}$ ) [23].

Studies into peripheral airway function in A-T are lacking.

\section{Sleep-related breathing disorder}

As the neurological condition progresses and decreased pulmonary reserve develops in the late teens, sleep-related breathing problems may contribute to respiratory morbidity. McGRATH-Morrow et al. [45] performed overnight polysomnography on 12 adolescents with A-T at a median (range) age of 16 (1320) years. All subjects in the study were wheelchair-bound and the median (range) FVC was 44 (16-82)\% pred. The authors found that the majority of A-T adolescents unexpectedly had infrequent partial or complete upper airway obstructions during sleep and minimal night-time hypoxaemia. Subjects did have decreased sleep efficiency, probably due in part to their underlying neurological condition. No correlation was found between FVC and sleep efficiency in the A-T subjects, unlike subjects with cystic fibrosis where the magnitude of sleep disruption is associated with severity of lung disease (low FEV1), but is not directly correlated with the degree of nocturnal hypoxaemia or hypoventilation [46]. There are no studies on the use of noninvasive ventilation in A-T for sleep-related breathing disorder, but extrapolating from other neuromuscular diseases, if there is evidence of abnormal gas exchange or sleep fragmentation then supplemental oxygen or noninvasive ventilation support should be considered [44].

\section{Cough and mucociliary clearance}

Some aspects of lung disease seen in A-T may be attributable to progressive neurological decline. Coordinated and maximal inspiratory and expiratory efforts are needed to generate effective cough and to clear airway secretions. Breathing and coughing involve cyclic motor acts that require cerebellar coordination (which is impaired in A-T) and it is likely that disordered muscle control (in addition to muscle weakness) exacerbates the decline in respiratory function. Abnormal respiratory muscle function resulting from neurological decline can lead to decreased tidal volumes and ineffective cough [41]. A decline in maximal respiratory muscle function has been found to be a marker for disease progression and respiratory failure in A-T patients [41]. Clinical indicators of lung disease due to impairment of upper airway and respiratory muscle systems may include chronic weak or dyscoordinate cough and prolonged or difficult recovery from respiratory illnesses. Lack of conditioning in patients with A-T who are wheelchair-bound may contribute to the deterioration in lung function.

In children and adolescents with neuromuscular weakness, those with inspiratory vital capacity $<1.1 \mathrm{~L}$ were shown to be at increased risk of hospitalisation due to lower RTIs [44]. Hence, measurements of vital capacity can also be used as surrogate markers for respiratory muscle strength. From this standpoint, A-T patients are 
approached in much the same way as those with other diseases causing neuromuscular impairment, such as Duchenne muscular dystrophy (DMD) and spinal muscular atrophy, although incoordination and fatigue are more significant contributors to lung disease in A-T, in addition to muscle weakness.

Evaluation of maximum inspiratory and expiratory pressures (MIP and MEP, respectively) is a well-established marker of respiratory muscle strength and coordination [47].

Respiratory muscle strength training (RMST) focuses on increasing the force-generating capacity of the inspiratory and expiratory muscles [48] when disease progression leads to worsening respiratory status with dyscoordinate and impaired cough and mucociliary clearance (aspiration due to swallowing dysfunction). Very scant A-T-specific data are available, but a recent longitudinal study assessed spirometry, subjective sensation of dyspnoea, MIP, MEP and quality of life before and after a 24-week inspiratory muscle training programme in $11 \mathrm{~A}-\mathrm{T}$ patients and nine healthy volunteers. It was shown that inspiratory muscle training was effective in improving ventilatory pattern, lung volume, respiratory muscle strength and the health and vitality domains of quality of life in patients with A-T. Inspiratory muscle training may be an effective adjunct therapy to drug treatment for patients with A-T [49]. Whether or not swallow safety to reduce aspiration improves after RMST, as has been shown for some other neurodegenerative conditions was not evaluated in this study [50].

Mucociliary clearance is impaired following recurrent infections and aspiration, both commonly seen in A-T. The resulting mucosal damage and secondary ciliary dyskinesia further impairs secretion clearance. Airway clearance physiotherapy, as used in cystic fibrosis or primary ciliary dyskinesia may mobilise mucus in the lower airways. Measures to improve pulmonary clearance and prevent atelectasis are likely to be beneficial in A-T patients, especially those with more advanced neurological decline, as they have both impaired mucociliary and cough clearance. Again, this is extrapolation from evidence and experience from other chronic respiratory conditions, although even in these it cannot be said that the evidence base is stellar. Such interventions include the use of chest physiotherapy, flutter valve therapy and cough assist devices. As in other neuromuscular disease disorders, regimens may be increased during episodes of acute illness [51, 52]. A-T patients have significantly lower inspiratory volume prior to cough, FVC cough, peak cough flow, peak cough flow to peak expiratory flow ratio and number of spikes per manoeuvre than healthy controls [53]. Additionally, although peak cough flow increased with age, the yearly rate of increase was significantly lower than normal. Thus, A-T patients had a weak cough compared to healthy controls of similar ages and cough worsened with age [53]. Cough assist devices (I. Sarouk, The Edmond and Lily Safra Children's Hospital, Sheba Medical Center, Ramat-Gan, Israel; personal communication) were used with some success in a small study where three out of five patients showed significant improvement in FVC, peak cough flow and mid-expiratory flows.

Physiotherapy advice regarding which techniques to adopt to optimise airway clearance before irreversible structural lung damage occurs is desirable, although there is no firm evidence base to give specific advice for A-T. Some form of regular exercise, even in younger children who are still mobile and not yet on a downward trajectory of pulmonary and neurological decline, is usually advised to parents and carers by most experts who look after children with A-T.

There are no studies into the efficacy of nebulised hypertonic saline, rhDNase, or inhaled mannitol on mobilisation of mucus in A-T. In AT-patients in general, inhalation therapy is likely to be less effective because of the neurological aspects of the disease. Extrapolating from studies in other groups of patients (non-cystic fibrosis bronchiectasis or airway malacia), it is likely but not certain that rhDNase is probably ineffective. Hypertonic saline or inhaled mannitol may offer some benefit in some patients [54] and this is supported by anecdotes of $n=1$ clinical trials for these medications in carefully selected patients and the experience of experts who look after children with A-T.

\section{Genotype/phenotype correlation in A-T}

It has been known for some time that there is heterogeneity of disturbances of immunoregulatory mechanisms in A-T and FioriLli et al. [55] postulated that the presence or absence of the ATM protein best explained the clinical heterogeneity and immunodeficiency. More recent work has confirmed this and has shown that patients with mutations resulting in a demonstrated complete loss of ATM kinase activity were significantly more likely to suffer from recurrent respiratory infections and have a greater need for prophylactic antibiotics, compared with those with leaky splice site or missense mutations resulting in expression of either some normal or mutant residual kinase activity. These results suggested that small amounts of preserved ATM function protects against the development of immune deficiency [10, 11]. MICOL et al. [9] in a large French registry study classified mutations according to the expected functional activity of the ATM product as either complete loss of expression or activity (class A or null) or reduced expression or some residual activity (class B or hypomorphic). They found RTIs to be the main risk factor for mortality among patients with hypomorphic mutations in $A T M$, while those with null mutations have a higher risk for 
cancer (mainly haematological malignancies) at younger ages. They hypothesised that RTIs increased mortality in patients with a hypomorphic mutation compared with those with two null mutations; this could probably be explained by the later onset of cancer in the group with hypomorphic mutations [9]. However, it is important to note that in a large cohort of children with early clinical onset the ATM protein was absent, showing that the classical phenotype is present in most children with A-T [56].

\section{Nutritional effects on lung disease and immune function}

Several small cross-sectional studies have shown that patients with A-T exhibit high rates of malnutrition, short stature and reduced lean body mass [56-59] (E. Stewart, et al., Nottingham Children's Hospital, UK Paediatric National Clinic, Nottingham University Hospitals, NHS Trust, Nottingham, UK; personal communication). A high percentage (58\%) of A-T patients had a BMI below the third percentile, despite apparently adequate energy intake especially until early adolescence, and this may reflect underlying hypercatabolism due to oxidative stress and inflammation [57]. There is a progressive reduction in the BMI with increasing age [57, 59] (E. Stewart et al.; personal communication). The onset of malnutrition depends on the relationship between nutrient balance and nutrient requirement, and a patient with A-T either loses weight or fails to grow normally if their absorbed energy intake is less than their total daily energy expenditure. Numerous factors including neurodegeneration, limited food intake with progressive disease, dysphagia and/or swallowing incoordination, limited physical activity, hormonal changes, hypogonadism, insulin resistance, glucose intolerance, abnormal expression of insulin-like growth factor-1 (somatomedin C) and low levels of insulin-like growth factor binding protein-3, infections and an associated hypercatabolic state all contribute to poor growth [56, 60]. In cystic fibrosis, lung inflammation appears to be related to increases in resting metabolic rate. Acute exacerbations of the chronic lung disease increases resting metabolic rate, which returns to a basal level some weeks after the inflammation is treated [61]; similar factors are likely (but not proven) to be contributory in A-T. A-T causes extreme insulin resistance [62], but clinical diabetes seems to be diagnosed infrequently [63-66]; in a Turkish series of 160 patients somatic growth retardation was reported to be common, but only two patients had frank diabetes mellitus [67]. It is unclear whether the presence of diabetes also affects lung disease in any way, but diabetes should be borne in mind during peri-operative management (see later).

An anthropometric assessment based on simple measurements, such as weight and height, is insufficient to identify changes in body composition. Using more accurate assessment of body cell mass (BCM) by methods such as bioimpedance assessment [59] (Pommering, Dept of Nutrition and Dietetics, The Royal Brisbane \& Women's Hospital, Brisbane, Queensland, Australia; personal communication), the prevalence of malnutrition in A-T patients was found to be high (69\%). Of particular note, three children identified as overweight or obese using standard anthropometry were identified as malnourished $(n=2)$ or moderately BCM-depleted $(n=1)$, and BCM depletion worsened with age whereby all participants aged $>9$ years were identified as malnourished [59].

Worsening nutritional status increases infection-related morbidity and mortality [68].

In children, malnutrition is of particular concern since it adversely affects not only statural growth but may also impact lung development [69]. Gastrostomy may be required to improve the quality and quantity of nutrition, reduce aspiration-related risks and improve the quality of life related to mealtimes in people with A-T [70].

\section{Dyscoordinate swallowing, aspiration, gastro-oesophageal reflux and gastrostomy} Impairment in bulbar muscle function can lead to swallowing dysfunction and chronic aspiration, which can cause and exacerbate lung disease $[71,72]$. In A-T, dysphagic problems commonly emerge in the second decade of life [72]. However, unlike individuals with neuromuscular disorders such as DMD and spinal muscular atrophy, in patients with A-T the reason for aspiration is a dyscoordinate swallow that is multifactorial rather than due to primary muscle weakness. Ataxia, hyperkinesis and bulbar dysfunction due to cerebellar involvement all lead to dyscoordination of the oral motor and swallowing movements. In the experience of the task force members, unlike the neuromuscular dystrophy patients who have problems with thick consistencies because of a weak swallow [73], children with A-T are observed to have problems with thin consistencies, and this has been shown by videofluoroscopic swallow study (VFSS) evaluation [71], which reported that aspiration occurred most frequently when older patients were drinking thin liquids through a straw.

Cough or congestion with meals may suggest dysfunctional swallow and aspiration. Poor weight gain may be an indicator of excessive caloric expenditure due to the increased work of breathing or difficulty taking in adequate calories [56-58].

Fatigue, while not measurable or well delineated in clinical research, is characteristic for A-T patients and may further contribute to aspiration and inefficient cough. Oropharyngeal dysphagia with aspiration is common and appears to be progressive in older patients (second decade) with A-T. The onset of dysphagia appears to coincide with a decrease in nutritional status although in a cross-sectional study it was not 
possible to distinguish between nutritional deficiency as a cause or effect of the dysphagia [71]. Neither the presence nor absence of coughing during mealtimes was found to be a reliable indicator of airway contamination during swallowing, as silent aspiration occurred in $71 \%$ of the patients whose aspiration was detected on videofluoroscopy. VFSS is the gold standard for assessing potential abnormalities of the pharyngeal phase of swallowing, which can only be inferred from a clinical observation. Radiographic confirmation of swallowing dysfunction is often used for guiding treatment in the patient with A-T although there is no universal consensus among task force members regarding this and some rely only on clinical assessment including observation and neck auscultation during mealtimes. Judicious use of a VFSS protocol that has been developed to capture dysphagic patterns while limiting radiation exposure is recommended [71]. This was achieved by limiting the duration of the VFSS and studying only those older patients (mostly children aged $>9$ years) with A-T with clinical evidence of oropharyngeal dysphagia (e.g. coughing or choking during or between meals, excessive drooling or exceedingly long mealtimes defined as $>40$ min on average) and/or recurrent RTIs or an otherwise unexplained drop in weight percentile or loss of weight. Total videofluoroscopy time averaged $\sim 1$ min per patient (range 40-90 s). This corresponds to an estimated effective dose of $0.1 \mathrm{mSv}$ (10 mrem) (a typical chest radiograph delivers an effective dose of 0.02-0.04 mSv) [71]. To put this into perspective, the average annual dose of natural background radiation is $2.2 \mathrm{mSv}$ in the UK [74] or $\sim 3 \mathrm{mSv}$ per year at sea level and $6 \mathrm{mSv}$ at higher elevations, such as Denver, in the USA [75].

Regular reviews by a speech and language therapist are vital so that appropriate early advice for changes in feeding routine such as elimination of thin liquids, pacing to slow the rate of liquid intake and having easily chewable food, etc. can be made. Advice about body posture to maintain an upright stable position to avoid shortening of the neck during eating and drinking is crucial. Ongoing concerns regarding aspiration and/or adequacy of nutrition should prompt referral for gastrostomy insertion. In the LEFTON-GrEIF et al. [70] series, $16 \%$ of patients underwent gastrostomy placement for nonpalliative reasons. This retrospective study demonstrated that safe gastrostomy placement and caregiver satisfaction (easier mealtimes) can be achieved when patients with A-T have feeding tubes placed at younger ages. While it is difficult to define the optimal age for gastrostomy placement, significant progressive decline in weight, length and BMI z-scores appears to start from the age of 8 years onwards (E. Stewart et al.; personal communication). Patients with childhood onset of disease with predictable progression and impaired swallowing may benefit from early rather than late placement of a feeding tube [70]. Even though a significant increase in weight or BMI z-score may not be seen following gastrostomy insertion in A-T and other static or progressive severe neurological disorders, an almost universal improvement in health-related quality of life scores is reported [70, 76]. Ease of medication administration as well as feeding appears to have a positive impact on the child's health at 6 and 12 months in the majority [76]. Additionally, delayed insertion of a gastrostomy is more likely to be associated with worse outcomes [70, 77].

\section{Scoliosis in A-T}

There have been no systematic studies of the incidence and natural history of scoliosis in A-T, although older literature mentions that it is common [78]. Severe idiopathic scoliosis (6.7/1000) is also described among A-T blood relatives [79] and may have a similar explanation as increased breast cancer risk in A-T carriers. Data from the Nottingham clinic [21] show that significant scoliosis is present in 18 (22.5\%) out of 80 patients. The vast majority of patients with scoliosis have classical A-T; only two (11.1\%) out of 18 patients with scoliosis had atypical A-T. The age range at diagnosis of scoliosis is $5-19$ years, it is equally common among males and females and three (16.7\%) out of 18 patients had severe scoliosis with two requiring spinal surgery.

Thus scoliosis is more common in adolescents as neurological disease progresses and patients spend prolonged time in a wheelchair and have the tendency to lean on the same elbow, although it is not possible to say if there is a cause-effect relationship, reverse causation or a mere association. Scoliosis varies from mild to severe. In progressive neuromuscular disease it has been shown that scoliosis aggravates restrictive lung disease due to an imbalance between the "load" or altered respiratory mechanics and the "pump" or the respiratory muscle strength [80]. Maintaining assisted ambulation as long as possible (e.g. use of walkers instead of wheelchairs) might prevent scoliosis and further deterioration of lung function, but further studies are needed to sustain this clinical observation.

\section{Risks of diagnostic radiation in $\mathrm{A}-\mathrm{T}$}

Patients with all forms of A-T are unusually sensitive to therapeutic doses of ionising radiation [81]. In vitro, fibroblasts and lymphoblasts from A-T homozygotes show increased sensitivity to ionising radiation and a number of radiomimetic and free-radical producing agents [82, 83]. Increased cellular and chromosomal radiosensitivity forms the basis of one of the highly sensitive and reasonably specific diagnostic tests used for the diagnosis of A-T [84]. The available evidence for radiosensitivity in patients with A-T is based on observations of patients who have received radiotherapy for the treatment of cancers before the diagnosis of A-T was made. Exposure of children with A-T to therapeutic doses of ionising radiation has resulted in death as a result of severe adverse reaction to the radiotherapy. However, there are no data available regarding the 
adverse effects of diagnostic radiography or CT scans in patients with A-T. In the absence of any evidence a pragmatic approach is followed by most members of this task force for the use of diagnostic radiographic and CT scans. Hence a single radiograph can be performed for diagnostic purposes such as the evaluation of scoliosis because avoidance could result in delayed or missed diagnoses and necessary treatment. The clinician and radiologist should consider the risks and benefits of each study and minimise radiation exposure as much as possible; repeated radiography should be avoided, as even exposure to diagnostic X-rays in early life in the general population is associated with increased risk of childhood leukaemia [85].

CT scanning provides excellent evaluation of the lungs, but the disadvantage of CT is the radiation exposure, although technical innovations have enabled its reduction [86]. A single low-dose CT scan is sometimes used, especially if ILD is suspected. Because radiation dose is directly proportional to the amount of the body that is imaged, limiting CT scanning to a specific area of concern further reduces the dose. Where possible, the smallest radiation dose should be used. Nonradiation imaging studies such as MRI scans or thoracic ultrasound (the latter especially for pleural disease) are sometimes considered. MRI is not typically used for the lungs due to the inability to visualise the air-filled alveoli; however, a diseased lung, e.g. areas of collapse and consolidation, bronchiectasis and bronchial wall thickening [87] or with alveolar haemorrhage [88], can be seen on MRI as a nonspecific airspace-filling process, similar to findings on a CT scan, and may provide similar information to a CT scan without the increased radiation exposure; these modalities are increasingly used. MRI with diffusion weighted imaging has been shown to be a reliable technique to detect lung alterations in patients with various immunodeficiency diseases [89, 90]. CT scans and MRI findings were comparable for moderate-to-severe degrees of bronchial and parenchymal alterations. Even for A-T, it has been recently demonstrated for the first time that MRI can be valuable in the assessment of the extent and severity of pulmonary changes in children and adults and is likely to become the modality of choice in the future, especially if scan times can be shortened and resolution improved [91].

\section{Cancer in A-T}

A detailed discussion of cancer in A-T is beyond the scope of this article. $\sim 20 \%$ of A-T patients will develop a malignancy during their lifetime. The vast majority of these are of lymphoid origin. There is no consensus regarding the optimal strategy for treating children with $\mathrm{A}-\mathrm{T}$ who develop haematopoietic malignancies. Historically, many of these children have been treated with therapy that is much less intensive than the conventional approach for non-A-T patients with similar malignancies (treatment protocols utilising lower doses of radiation therapy and alternatives to radiomimetic drugs such as bleomycin and neurotoxic agents, and modified chemotherapy protocols to avoid drugs that carry a high risk of the child developing a second malignancy (topoisomerase inhibitors)). Although these less intensive approaches may have stemmed from perceptions that these children would not tolerate intensive therapy, there are in fact no data to support this view [21]. However, it is clear that children with A-T require a modification in certain components of intensive therapy [21].

\section{Perioperative management of a patient with A-T undergoing surgery}

Patients with A-T may require general anaesthesia for bronchoscopy and bronchoalveolar lavage, lung biopsy, gastrostomy insertion or scoliosis surgery. There have been scant evidence to date on the anaesthetic risks in A-T, and a full evaluation should be performed prior to anaesthesia [92, 93]. A protocol for careful assessment in high-risk ventilator-dependent children with predominantly neuromuscular disorders allowed operative intervention to be performed under general anaesthesia, minimised post-operative complications and was associated with a good outcome in the majority [94]. A similar detailed evaluation is undertaken in patients with A-T by most experts managing these patients. Patients with A-T are at increased risk of aspiration and some may require post-operative ventilatory support. A-T can be associated with impaired insulin secretion, increased risk of insulin resistance, glucose intolerance and type 2 diabetes [62, 95, 96]. Peri-operative serum glucose control monitoring is important with the instigation of appropriate treatment. Use of succinylcholine may cause hyperkalaemia in patients with significant neuropathy and muscle weakness. The largest series describes $21 \mathrm{~A}-\mathrm{T}$ patients who underwent 34 episodes of general anaesthesia for a total of 41 procedures over a 15-year period (1995-2009). Anaesthetic risk was comparable to that for other medically complex paediatric patients. All patients were seen in a multidisciplinary centre, allowing for disease-specific peri-operative optimisation and post-operative follow-up. All survived and there were no complications of anaesthesia and no unexpected admissions to the paediatric intensive care unit. A quarter of the patients required supplemental oxygen for up to $24 \mathrm{~h}$ post-operatively. All patients were anaesthetised within an inpatient hospital setting, even though $20 \%$ were able to go home on the day of surgery. Paediatric intensive care has been shown to be beneficial for stabilisation and optimisation, especially for emergency procedures [97].

\section{Organisation of care for lung disease in A-T}

Respiratory disease in A-T is complex and its understanding and management requires multidisciplinary input. Current approaches are based on minimal best available evidence in A-T, and extrapolation from 
other more common disorders such as cystic fibrosis. The lung disease of A-T is similar in many ways to cystic fibrosis in terms of the exacerbation of lung disease and the sequelae (chronic pulmonary sepsis, bronchiectasis and chronic rhinosinusitis) [23]. Recognising this similarity to patients with cystic fibrosis, the early involvement of a respiratory physician is desirable to monitor lung function and optimise chest care, including early aggressive antibiotic treatment, good airway clearance techniques and smoking cessation advice including avoidance of second-hand smoke [23]. However, the neurological and immunological contributions to lung disease in A-T are unlike those in cystic fibrosis and this emphasises the need for multidisciplinary expertise to manage this devastating disease.

Several multidisciplinary clinics across Europe (the UK, Netherlands, Norway, France and Germany) and elsewhere (Israel, Baltimore (MD, USA) and Brisbane (Australia)) manage patients with A-T. These clinics provide multidisciplinary expertise for this rare multisystem disease.

Collaboration between these clinics and the establishment of a multinational patient registry and prospective studies will help determine the outcomes of various prophylactic antibiotic regimes, the effectiveness of regular versus as-required, and oral versus i.v. antibiotic treatment in A-T. It is hoped that such collaborations will allow the performance of randomised controlled trials and an improved evidence base for the disease.

\section{Current practice}

\section{Immunology and infections}

1) Routine immunological investigations for each patient, including total lg levels and subclasses, specific IgG antibodies to tetanus, Haemophilus influenzae B and pneumococcal serotypes and immunophenotyping of peripheral blood.

2) Since the risks imposed by the development of vaccine-preventable illnesses are potentially fatal, patients with ataxia telangectasia (A-T) receive all available immunisations to respiratory pathogens (including pneumococcal polysaccharide vaccine following pneumococcal conjugate vaccinel and inactivated annual influenza vaccine.

3) A clinical history of susceptibility to infections is considered as more than three respiratory tract infections (RTIs) per winter season requiring antibiotics or a severe/atypical response to a single infection. Early aggressive treatment with antibiotics and airway clearance is instituted.

4) If recurrent RTIs present later in life, other contributory factors le.g. cough/swallowing difficulties, neuromuscular abnormalities, underlying interstitial lung disease (ILD)) are investigated aggressively.

5) Prophylactic antibiotic treatment is considered when the burden of respiratory infections is high, if there is associated specific polysaccharide antibody deficiency and when there is established bronchiectasis. Often the decision is based on the judgement of the caretakers, patient and physician. The advantages (shorter duration of illness and better quality of life) are weighed against the disadvantages (microbiological resistance and side-effects).

6) Immune replacement therapy is considered when hypogammaglobulinemia and/or specific antibody deficiency is present together with a history of recurrent respiratory infections or established bronchiectasis, or when other preventive measures such as vaccinations and prophylactic antibiotics do not result in clinical improvement of the RTI symptoms irrespective of the severity of the immunological abnormalities.

7) Regular surveillance microbiology on respiratory samples is undertaken and early aggressive treatment initiated.

\section{Respiratory monitoring}

8) Baseline spirometry is attempted in children who are old enough to perform tests. Regular assessment of lung function can provide valuable information about subtle progression of lung disease, even in the absence of clinical symptoms. This is also performed during respiratory illnesses and for peri-operative assessment and early interventions. Regular assessments at least twice a year provide an impression of the degree of lung decline and allows for proactive early intervention.

9) Peak cough flow rate as well as forced vital capacity manoeuvres, are considered a mainstay in the clinical assessment of $A-T$ patients.

10) Regular airway clearance and measures to augment cough and mucociliary clearance is advised by most experts looking after children with A-T. This is stepped up if respiratory symptoms worsen. Use of nebulised hypertonic saline or inhaled mannitol may be a useful adjunct to airway clearance in some patients.

11) Overnight polysomnography is considered in adolescents with A-T, particularly in those in whom there is a clinical suspicion of sleep-related breathing abnormalities, rapid or progressive decline in lung function or who are developing scoliosis.

12) Progressive, diffuse lung disease that poorly responds to aggressive antibiotic therapy, physiotherapy and immunoglobulin replacement and to which aspiration does not seem to contribute should lead to consideration of noninfective causes of lung disease in A-T, including ILD and pulmonary fibrosis and early treatment with corticosteroids. 
Current practice Continued

\section{Nutrition and swallow}

13) Assessment of the adequacy and safety of nutritional intake should be performed at least annually. Provision of supplemental calories by the oral or enteral route should be undertaken in those with worsening nutritional status. Early gastrostomy insertion is likely to be required in those with unsafe swallow or inadequate nutritional intake. Gastrostomy placement is considered when patients with A-T are young and begin to present with progressive nutritional insufficiency, respiratory deterioration and aspiration secondary to dysphagia that are unresponsive to common conservative measures (e.g. dietary modifications and medical therapies) or when feeding disrupts activities of daily living.

\section{Miscellaneous}

14) Early and continued physiotherapy often mitigates the occurrence of and effects of contractures and scoliosis. Chest wall bracing is often used as a temporising, but never curative, procedure, but can have a negative impact on vital capacity and can decrease both respiratory system compliance and tidal ventilation. Spinal stabilisation using a variety of different surgical techniques has been the standard approach for treating scoliosis in patients with neuromuscular disease and scoliosis; however, timing of surgical intervention needs to be individually determined by regular and proactive surveillance.

15) Multidisciplinary disease-specific peri-operative evaluation with optimisation of pulmonary function and follow-up should be ensured to improve outcomes.

16) Although healthcare models will vary in different countries, multidisciplinary "one-stop" service provision by all subspecialities should be the target in this rare devastating illness.

\section{References}

1 Orphanet. Ataxia-telangiectasia. www.orpha.net/consor/cgi-bin/OC_Exp.php?lng=EN\&Expert=100 Date last accessed: March 15, 2015. Date last updated: October 2007.

2 Woods CG, Bundey SE, Taylor AM. Unusual features in the inheritance of ataxia telangiectasia. Hum Genet 1990; 84: $555-562$.

3 Thompson D, Duedal S, Kirner J, et al. Cancer risks and mortality in heterozygous ATM mutation carriers. J Natl Cancer Inst 2005; 97: 813-822.

4 Gathmann B, Binder N, Ehl S, et al. The European internet-based patient and research database for primary immunodeficiencies: update 2011. Clin Exp Immunol 2012; 167: 479-491.

5 Verhagen MM, Last JI, Hogervorst FB, et al. Presence of ATM protein and residual kinase activity correlates with the phenotype in ataxia-telangiectasia: a genotype-phenotype study. Hum Mutat 2012; 33: 561-571.

6 Teive HAG, Moro A, Moscovich M, et al. Ataxia-telangiectasia - a historical review and a proposal for a new designation: ATM syndrome. J Neurol Sci 2015; 355: 3-6.

$7 \quad$ Morrell D, Cromartie E, Swift M. Mortality and cancer incidence in 263 patients with ataxia-telangiectasia. J Natl Cancer Inst 1986; 77: 89-92.

8 Crawford TO, Skolasky RL, Fernandez R, et al. Survival probability in ataxia telangiectasia. Arch Dis Child 2006; 91: 610-611.

9 Micol R, Ben Slama L, Suarez F, et al. Morbidity and mortality from ataxia-telangiectasia are associated with ATM genotype. J Allergy Clin Immunol 2011; 128: 382-389.

10 Staples ER, McDermott EM, Reiman A, et al. Immunodeficiency in ataxia telangiectasia is correlated strongly with the presence of two null mutations in the ataxia telangiectasia mutated gene. Clin Exp Immunol 2008; 153: 214-220.

11 Driessen GJ, Ijspeert $\mathrm{H}$, Weemaes CM, et al. Antibody deficiency in patients with ataxia telangiectasia is caused by disturbed B- and T-cell homeostasis and reduced immune repertoire diversity. J Allergy Clin Immunol 2013; 131: $1367-1375$

12 Nowak-Wegrzyn A, Crawford TO, Winkelstein JA, et al. Immunodeficiency and infections in ataxia-telangiectasia. J Pediatr 2004; 144: 505-511.

13 Noordzij JG, Wulffraat NM, Haraldsson A, et al. Ataxia-telangiectasia patients presenting with hyper-IgM syndrome. Arch Dis Child 2009; 94: 448-449.

14 Waldmann TA, Broder S, Goldman CK, et al. Disorders of B cells and helper T cells in the pathogenesis of the immunoglobulin deficiency of patients with ataxia telangiectasia. J Clin Invest 1983; 71: 282-295.

15 Oxelius VA, Berkel AI, Hanson LA. IgG2 deficiency in ataxia-telangiectasia. N Engl J Med 1982; 306: 515-517.

16 Davies EG. Update on the management of the immunodeficiency in ataxia-telangiectasia. Expert Rev Clin Immunol 2009; 5: 565-575.

17 Schroeder SA, Zielen S. Infections of the respiratory system in patients with ataxia-telangiectasia. Pediatr Pulmonol 2014; 49: 389-399.

18 Sanal O, Ersoy F, Yel L, et al. Impaired IgG antibody production to pneumococcal polysaccharides in patients with ataxia-telangiectasia. J Clin Immunol 1999; 19: 326-334.

19 Stray-Pedersen A, Aaberge IS, Früh A, et al. Pneumococcal conjugate vaccine followed by pneumococcal polysaccharide vaccine; immunogenicity in patients with ataxia-telangiectasia. Clin Exp Immunol 2005; 140: 507-516.

20 Schubert R, Reichenbach J, Rose M, et al. Immunogenicity of the seven valent pneumococcal conjugate vaccine in patients with ataxia-telangiectasia. Pediatr Infect Dis J 2004; 23: 269-270.

21 AT Society. Ataxia-telangiectasia in Children: Guidance on Diagnosis and Clinical Care. www.atsociety.org.uk/ clinical-guidance Date last accessed: March 17, 2015. Date last updated: September 9, 2015.

22 Moin M, Aghamohammadi A, Kouhi A, et al. Ataxia-telangiectasia in Iran: clinical and laboratory features of 104 patients. Pediatr Neurol 2007; 37: 21-28.

23 Bott L, Lebreton J, Thumerelle C, et al. Lung disease in ataxia-telangiectasia. Acta Paediatr 2007; 96: 1021-1024.

24 Bhatt JM, Bush A. Microbiological surveillance in lung disease in ataxia telangiectasia. Eur Respir J 2014; 43: 1797-1801. 
Ammann AJ, Good RA, Bier D, et al. Long-term plasma infusions in a patient with ataxia-telangiectasia and deficient IGA and IGE. Pediatrics 1969; 44: 672-676.

Cawley LP, Schenken JR. Monoclonal hypergammaglobulinemia of the gamma M type in a nine-year-old girl with ataxia-telangiectasia. Am J Clin Pathol 1970; 54: 790-801.

Chopra C, Davies G, Taylor M, et al. Immune deficiency in ataxia-telangiectasia: a longitudinal study of 44 patients. Clin Exp Immunol 2014; 176: 275-282.

McGrath-Morrow SA, Lederman HM, Aherrera AD, et al. Pulmonary function in children and young adults with ataxia telangiectasia. Pediatr Pulmonol 2014; 49: 84-90.

Borte M, Pac M, Serban M, et al. Efficacy and safety of Hizentra ${ }^{\circ}$, a new $20 \%$ immunoglobulin preparation for subcutaneous administration, in pediatric patients with primary immunodeficiency. J Clin Immunol 2011; 31: 752-761.

Jolles S, Bernatowska E, de Gracia J, et al. Efficacy and safety of Hizentra in patients with primary immunodeficiency after a dose-equivalent switch from intravenous or subcutaneous replacement therapy. Clin Immunol 2011; 141: 90-102. Chapel HM, Spickett GP, Ericson D, et al. The comparison of the efficacy and safety of intravenous versus subcutaneous immunoglobulin replacement therapy. J Clin Immunol 2000; 20: 94-100.

Schroeder SA, Swift M, Sandoval C, et al. Interstitial lung disease in patients with ataxia-telangiectasia. Pediatr Pulmonol 2005; 39: 537-543.

Rasheed N, Wang X, Niu Q-T, et al. Atm-deficient mice: an osteoporosis model with defective osteoblast differentiation and increased osteoclastogenesis. Hum Mol Genet 2006; 15: 1938-1948.

Davis T, Kipling D. Assessing the role of stress signalling via p38 MAP kinase in the premature senescence of ataxia telangiectasia and Werner syndrome fibroblasts. Biogerontology 2009; 10: 253-266.

Canny GJ, Roifman C, Weitzman S, et al. A pulmonary infiltrate in a child with ataxia telangiectasia. Ann Allergy 1988; 61: 422-423, 466-468.

Yalçin B, Kutluk MT, Sanal O, et al. Hodgkin's disease and ataxia telangiectasia with pulmonary cavities. Pediatr Pulmonol 2002; 33: 399-403.

Chen RL, Wang PJ, Hsu YH, et al. Severe lung fibrosis after chemotherapy in a child with ataxia-telangiectasia. J Pediatr Hematol Oncol 2002; 24: 77-79.

Sandoval C, Swift M. Treatment of lymphoid malignancies in patients with ataxia-telangiectasia. Med Pediatr Oncol 1998; 31: 491-497.

Taylor AM, Rosney CM, Campbell JB. Unusual sensitivity of ataxia telangiectasia cells to bleomycin. Cancer Res 1979; 39: 1046-1050.

Tangsinmankong N, Wayne AS, Howenstine MS, et al. Lymphocytic interstitial pneumonitis, elevated IgM concentration, and hepatosplenomegaly in ataxia-telangiectasia. J Pediatr 2001; 138: 939-941.

McGrath-Morrow S, Lefton-Greif M, Rosquist $\mathrm{K}$, et al. Pulmonary function in adolescents with ataxia telangiectasia. Pediatr Pulmonol 2008; 43: 59-66.

Berkun Y, Vilozni D, Levi Y, et al. Reversible airway obstruction in children with ataxia telangiectasia. Pediatr Pulmonol 2010; 45: 230-235.

3 Vilozni D, Berkun Y, Levi Y, et al. The feasibility and validity of forced spirometry in ataxia telangiectasia. Pediatr Pulmonol 2010; 45: 1030-1036.

McGrath-Morrow SA, Gower WA, Rothblum-Oviatt C, et al. Evaluation and management of pulmonary disease in ataxia-telangiectasia. Pediatr Pulmonol 2010; 45: 847-859.

McGrath-Morrow SA, Sterni L, McGinley B, et al. Polysomnographic values in adolescents with ataxia telangiectasia. Pediatr Pulmonol 2008; 43: 674-679.

Naqvi SK, Sotelo C, Murry L, et al. Sleep architecture in children and adolescents with cystic fibrosis and the association with severity of lung disease. Sleep Breath 2008; 12: 77-83.

Jackson CE, Rosenfeld J, Moore DH, et al. A preliminary evaluation of a prospective study of pulmonary function studies and symptoms of hypoventilation in ALS/MND patients. J Neurol Sci 2001; 191: 75-78.

Sapienza C, Troche M, Pitts T, et al. Respiratory strength training: concept and intervention outcomes. Semin Speech Lang 2011; 32: 21-30.

Félix E, Gimenes AC, Costa-Carvalho BT. Effects of inspiratory muscle training on lung volumes, respiratory muscle strength, and quality of life in patients with ataxia telangiectasia. Pediatr Pulmonol 2014; 49: 238-244.

Troche MS, Okun MS, Rosenbek JC, et al. Aspiration and swallowing in Parkinson disease and rehabilitation with EMST: a randomized trial. Neurology 2010; 75: 1912-1919.

Hull J, Aniapravan R, Chan E, et al. British Thoracic Society guideline for respiratory management of children with neuromuscular weakness. Thorax 2012; 67: Suppl. 1, i1-i40.

Finder JD, Birnkrant D, Carl J, et al. Respiratory care of the patient with Duchenne muscular dystrophy: ATS consensus statement. Am J Respir Crit Care Med 2004; 170: 456-465.

Vilozni D, Lavie M, Berkun Y, et al. Cough flow volume profile in ataxia telangiectasia. Eur Respir J 2011; 38: Suppl. 55, p2016.

4 Hart A, Sugumar K, Milan SJ, et al. Inhaled hyperosmolar agents for bronchiectasis. Cochrane Database Syst Rev 2014; 5: CD002996.

Fiorilli M, Businco L, Pandolfi F, et al. Heterogeneity of immunological abnormalities in ataxia-telangiectasia. J Clin Immunol 1983; 3: 135-141.

6 Voss S, Pietzner J, Hoche F, et al. Growth retardation and growth hormone deficiency in patients with ataxia telangiectasia. Growth Factors 2014; 32: 123-129.

Schubert R, Reichenbach J, Zielen S. Growth factor deficiency in patients with ataxia telangiectasia. Clin Exp Immunol 2005; 140: 517-519.

da Silva R, dos Santos-Valente EC, Burim Scomparini F, et al. The relationship between nutritional status, vitamin A and zinc levels and oxidative stress in patients with ataxia-telangiectasia. Allergol Immunopathol 2014; 42: 329-335.

Ross LJ, Capra S, Baguley B, et al. Nutritional status of patients with ataxia-telangiectasia: a case for early and ongoing nutrition support and intervention. J Paediatr Child Health 2015; 51: 802-807.

Peretz S, Jensen R, Baserga R, et al. ATM-dependent expression of the insulin-like growth factor-I receptor in a pathway regulating radiation response. Proc Natl Acad Sci USA 2001; 98: 1676-1681.

Pencharz PB, Durie PR. Pathogenesis of malnutrition in cystic fibrosis, and its treatment. Clin Nutr 2000; 19: 387-394. 
62 Bar RS, Levis WR, Rechler MM, et al. Extreme insulin resistance in ataxia telangiectasia: defect in affinity of insulin receptors. N Engl J Med 1978; 298: 1164-1171.

63 Schalch DS, McFarlin DE, Barlow MH. An unusual form of diabetes mellitus in ataxia telangiectasia. $N$ Engl $J$ Med 1970; 282: 1396-1402.

64 Morrell D, Chase CL, Kupper LL, et al. Diabetes mellitus in ataxia-telangiectasia, Fanconi anemia, xeroderma pigmentosum, common variable immune deficiency, and severe combined immune deficiency families. Diabetes 1986; 35: 143-147.

65 Robinson S, Kessling A. Diabetes secondary to genetic disorders. Baillières Clin Endocrinol Metab 1992; 6: 867-898.

66 Blevins LS, Gebhart SS. Insulin-resistant diabetes mellitus in a black woman with ataxia-telangiectasia. South Med J 1996; 89: 619-621.

67 Ersoy F, Berkel AI, Sanal O, et al. Twenty-year follow-up of 160 patients with ataxia-telangiectasia. Turk J Pediatr 1991; 33: 205-215.

68 Bresnahan KA, Tanumihardjo SA. Undernutrition, the acute phase response to infection, and its effects on micronutrient status indicators. Adv Nutr 2014; 5: 702-711.

69 Girardet JP, Viola S. Nutrition and severe chronic respiratory diseases: pathophysiologic mechanisms. Pediatr Pulmonol 2001; Suppl. 23: 20-21.

70 Lefton-Greif MA, Crawford TO, McGrath-Morrow S, et al. Safety and caregiver satisfaction with gastrostomy in patients with ataxia telangiectasia. Orphanet J Rare Dis 2011; 6: 23.

71 Lefton-Greif MA, Crawford TO, Winkelstein JA, et al. Oropharyngeal dysphagia and aspiration in patients with ataxia-telangiectasia. J Pediatr 2000; 136: 225-231.

72 Crawford TO, Mandir AS, Lefton-Greif MA, et al. Quantitative neurologic assessment of ataxia-telangiectasia. Neurology 2000; 54: 1505-1509.

73 van den Engel-Hoek L, Erasmus CE, van Hulst KC, et al. Children with central and peripheral neurologic disorders have distinguishable patterns of dysphagia on videofluoroscopic swallow study. J Child Neurol 2014; 29: 646-653.

74 UK Government. UK Atmospheric Nuclear Weapons Tests Factsheet 4: Radiation and Health. www.gov.uk/ government/uploads/system/uploads/attachment_data/file/82780/ntvfactsheet4.pdf Date last accessed: September 9, 2015. Date last updated: June 16, 2008.

75 Brody AS, Frush DP, Huda W, et al. Radiation risk to children from computed tomography. Pediatrics 2007; 120 $677-682$.

76 Mahant S, Friedman JN, Connolly B, et al. Tube feeding and quality of life in children with severe neurological impairment. Arch Dis Child 2009; 94: 668-673.

77 Sy K, Mahant S, Taback N, et al. Enterostomy tube placement in children with spinal muscular atrophy type 1 . I Pediatr 2006; 149: 837-839.

78 Sedgwick RP, Boder E. Ataxia-telangiectasia. In: Vinken PJ, Bruyn GW, eds. Handbook of Clinical Neurology. New York, American, 1972; pp. 267-339.

79 Welshimer K, Swift M. Congenital malformations and developmental disabilities in ataxia-telangiectasia, Fanconi anemia, and xeroderma pigmentosum families. Am J Hum Genet 1982; 34: 781-793.

80 Mayer OH. Scoliosis and the impact in neuromuscular disease. Paediatr Respir Rev 2015; 16: 35-42.

81 Pollard JM, Gatti RA. Clinical radiation sensitivity with DNA repair disorders: an overview. Int J Radiat Oncol Biol Phys 2009; 74: 1323-1331.

82 Shiloh Y, Tabor E, Becker Y. Cells from patients with ataxia telangiectasia are abnormally sensitive to the cytotoxic effect of a tumor promoter, phorbol-12-myristate-13-acetate. Mutat Res 1985; 149: 283-286.

83 Taylor AM, Laher HB, Morgan GR. Unscheduled DNA synthesis induced by streptonigrin in ataxia telangiectasia fibroblasts. Carcinogenesis 1985; 6: 945-947.

84 Huo YK, Wang Z, Hong JH, et al. Radiosensitivity of ataxia-telangiectasia, X-linked agammaglobulinemia, and related syndromes using a modified colony survival assay. Cancer Res 1994; 54: 2544-2547.

85 Bartley K, Metayer C, Selvin S, et al. Diagnostic X-rays and risk of childhood leukaemia. Int J Epidemiol 2010; 39: 1628-1637.

86 Wielpütz MO, Heußel CP, Herth FJF, et al. Radiological diagnosis in lung disease: factoring treatment options into the choice of diagnostic modality. Dtsch Ärztebl Int 2014; 111: 181-187.

87 Puderbach M, Eichinger M, Gahr J, et al. Proton MRI appearance of cystic fibrosis: comparison to CT. Eur Radiol 2007; 17: 716-724

88 Kaleel M, Schramm C, Pascal M, et al. Serial lung magnetic resonance imaging to monitor disease progression in a child with a diffuse alveolar hemorrhage syndrome. J Clin Med Res 2015; 7: 267-269.

89 Serra G, Milito C, Mitrevski M, et al. Lung MRI as a possible alternative to CT scan for patients with primary immune deficiencies and increased radiosensitivity. Chest 2011; 140: 1581-1589.

90 Milito C, Pulvirenti F, Serra G, et al. Lung magnetic resonance imaging with diffusion weighted imaging provides regional structural as well as functional information without radiation exposure in primary antibody deficiencies. $J$ Clin Immunol 2015; 35: 491-500.

91 Montella S, Mollica C, Finocchi A, et al. Non invasive assessment of lung disease in ataxia telangiectasia by high-field magnetic resonance imaging. J Clin Immunol 2013; 33: 1185-1191.

92 Baum VC. Anesthesia for Genetic, Metabolic, and Dysmorphic Syndromes of Childhood. 3rd Edn. Philadelphia, Wolters Kluwer, 2015.

93 Bissonnette B, ed. Syndromes: Rapid Recognition and Perioperative Management. 1st Edn. New York, McGraw-Hill, 2005.

94 Chatwin M, Bush A, Macrae DJ, et al. Risk management protocol for gastrostomy and jejunostomy insertion in ventilator dependent infants. Neuromuscul Disord 2013; 23: 289-297.

95 Miles PD, Treuner K, Latronica M, et al. Impaired insulin secretion in a mouse model of ataxia telangiectasia. Am J Physiol Endocrinol Metab 2007; 293: E70-E74.

96 Armata HL, Golebiowski D, Jung DY, et al. Requirement of the ATM/p53 tumor suppressor pathway for glucose homeostasis. Mol Cell Biol 2010; 30: 5787-5794.

97 Lockman JL, Iskander AJ, Bembea M, et al. Anesthetic and perioperative risk in the patient with ataxia-telangiectasia. Paediatr Anaesth 2012; 22: 256-262. 